\title{
Improved mobility models for charge transport in graphene
}

\author{
G. Nastasi ${ }^{1 *}$, V. Romano ${ }^{1}$ \\ ${ }^{1}$ Department of Mathematics and Computer Science, University of Catania, Italy \\ *Email address for correspondence: g.nastasi@unict.it \\ Communicated by Giorgio Fotia \\ Received on 12 10, 2018. Accepted on 02 15, 2019.
}

\begin{abstract}
Charge transport in graphene is crucial for the design of a new generation of nanoscale electron devices. A reasonable model is represented by the semiclassical Boltzmann equations for electrons in the valence and conduction bands. As shown by Romano et al. (J. Comput. Phys., 2015), the discontinuous Galerkin methods are a viable way to tackle the problem of the numerical integration of these equations, even if efficient DSMC with a proper inclusion of the Pauli principle have been also devised. One of the advantages of the solutions obtained with deterministic approach is of course the absence of statistical noise. This fact is crucial for an accurate estimation of the low field mobility as proved by Majorana et al. (J. Math. Industry, 2016) in the case of a unipolar charge transport in a suspended graphene sheet under a constant electric field.

The mobility expressions are essential for the drift-diffusion equations which constitute the most adopted models for charge transport in CAD. Here the analysis by Majorana et al. (J. Math. Industry, 2016) is improved in two ways: by including the charge transport both in the valence and conduction bands; by taking into account the presence of an oxide as substrate for the graphene sheet. New models of mobility are obtained and, in particular, relevant improvements of the low field mobility are achieved.
\end{abstract}

Keywords: Graphene; charge transport; mobility model; discontinuous Galerkin methods.

AMS subject classification: 82C70, 82D37, 65M60

\section{Introduction}

Graphene, a single layer of carbon atoms arranged into a honeycomb lattice, is considered as the ideal candidate for the ultimate generation of ultra integrated circuits. In fact, replacement of traditional semiconductors with graphene could allow the fabrication of devices, e.g. MOSFETs, whose channels (the active part) having a width of just a single atom, reaching in this way the theoretical limit of miniaturisation. For such a reason, an accurate description of charge transport in graphene is crucial. A reasonable model is represented by the semiclassical Boltzmann equations for electrons in the valence and conduction bands. As shown in [1], a viable way to tackle the problem of the numerical solutions of these equations is the discontinuous Galerkin method, even if efficient DSMC with a proper inclusion of the Pauli principle have been also devised [1]. One of the advantages of the solutions obtained with a deterministic approach, with respect to those obtained with DSMC, is of course the absence of statistical noise. This fact is crucial for an accurate estimation of the low field mobility as proved in [2] in the case of a unipolar charge transport in a suspended graphene sheet under a constant electric field.

The mobility expressions are essential for the drift-diffusion equations which constitute the most adopted models for charge transport in CAD. Here the analysis in [2] is improved in two ways: by including the charge transport both in the valence and conduction bands, and by taking into account the presence of an oxide as substrate for the graphene sheet. New models of mobility are obtained and, in particular, relevant improvements of the low field mobility are achieved.

The plan of the paper is as follows. In Section 2 the main features of the semiclassical charge transport in graphene are sketched and in Section 3 a Discontinuous Galerkin (DG) method is outlined for the numerical integration of the transport equations [3]. A new mobility model is presented in Section 4 in the case of suspended monolayer graphene and comparisons with other models already existing in the 
literature are performed. In the last section a further extension is presented for graphene on a substrate made of silicon dioxide.

\section{The semiclassical charge transport in graphene}

In a semiclassical kinetic setting, the charge transport in graphene is described, in general, by four Boltzmann equations, one for electrons in the valence $(\pi)$ band and one for electrons in the conduction $\left(\pi^{*}\right)$ band, that in turn can belong to the $K$ or $K^{\prime}$ valley. By assuming the $K$ and $K^{\prime}$ valleys as equivalent, then we can consider only the two equations

$$
\frac{\partial f_{s}}{\partial \mathrm{t}}+\mathbf{v}_{s} \cdot \nabla_{\mathbf{x}} f_{s}-\frac{e}{\hbar} \mathbf{E} \cdot \nabla_{\mathbf{k}} f_{s}=Q\left(f_{s}, f_{-s}\right) \quad(s= \pm 1)
$$

where $f_{s}=f_{s}(\mathrm{t}, \mathbf{x}, \mathbf{k})$ represents the distribution function of charge carriers in the conduction band (CB), for $s=1$, or in the valence band (VB), for $s=-1$, at position $\mathbf{x}$, time $t$ and wave-vector $\mathbf{k}$. We denote by $\nabla_{\mathbf{x}}$ and $\nabla_{\mathbf{k}}$ the gradients with respect to the position and wave vector, respectively. In the sequel, for simplifying the notation, we write also $f_{+}$and $f_{-}$to indicate the distributions of electrons in the conduction and valence bands. The group velocity $\mathbf{v}_{s}$ is related to the energy band $\varepsilon_{s}$ by

$$
\mathbf{v}_{s}=\frac{1}{\hbar} \nabla_{\mathbf{k}} \varepsilon_{s} .
$$

With a very good approximation [4] a linear dispersion relation holds for the energy bands $\varepsilon_{s}$ around the Dirac points; so that, choosing the origin of the reference frame in the $\mathbf{k}$-space coinciding with a Dirac point, we have

$$
\varepsilon_{s}=s \hbar v_{F}|\mathbf{k}|
$$

where $v_{F}$ is the (constant) Fermi velocity and $\hbar$ the Planck constant divided by $2 \pi$. The elementary (positive) charge is denoted by $e$. Here the electric field $\mathbf{E}$ is assumed as external, and therefore we do not include the Poisson equation. The right hand side of Equation (1) is the collision term representing the interaction of electrons with acoustic, optical and $K$ phonons. Acoustic phonon scattering is intravalley and intraband. Optical phonon scattering is intravalley and can be both longitudinal (LO) and transversal (TO). It can be intraband, that is it leaves electrons in the same band, or interband pushing electrons from an initial band to the other one. Scattering with optical phonon of type $K$ pushes electrons from a valley to a neighbor one (intervalley scattering). We assume that phonons are at thermal equilibrium. Hence, the general form of the collision term can be written as

$$
\begin{aligned}
Q\left(f_{s}, f_{-s}\right)= & \sum_{s^{\prime}}\left[\int_{\mathbb{R}^{2}} S_{s^{\prime}, s}\left(\mathbf{k}^{\prime}, \mathbf{k}\right) f_{s^{\prime}}\left(\mathrm{t}, \mathbf{x}, \mathbf{k}^{\prime}\right)\left(1-f_{s}(\mathrm{t}, \mathbf{x}, \mathbf{k})\right) d \mathbf{k}^{\prime}\right. \\
& \left.-\int_{\mathbb{R}^{2}} S_{s, s^{\prime}}\left(\mathbf{k}, \mathbf{k}^{\prime}\right) f_{s}(\mathrm{t}, \mathbf{x}, \mathbf{k})\left(1-f_{s^{\prime}}\left(\mathrm{t}, \mathbf{x}, \mathbf{k}^{\prime}\right)\right) d \mathbf{k}^{\prime}\right]
\end{aligned}
$$

where the total transition rate is given by the sum of the contributions of the several types of scatterings. $S_{s, s^{\prime}}$ represents the scattering involving the band $s$ and $s^{\prime}$ and it is in turn given by the sum of several type of electron-phonon interactions. More details can be found in $[1,3]$.

At the equilibrium the distribution function of electrons in both bands is the Fermi-Dirac distribution, given by

$$
f_{F D}^{ \pm}(\mathrm{t}, \mathbf{x}, \mathbf{k})=\frac{1}{1+\exp \left(\frac{\varepsilon_{ \pm}-\varepsilon_{F}}{k_{B} T}\right)},
$$

where $\varepsilon_{F}$ is the Fermi energy and the sign \pm indicates the CB $(+)$ or the VB $(-)$. The Fermi energy is related to the density. For example at equilibrium for the electrons in the conduction band the density is given by

$$
n=\frac{2}{(2 \pi)^{2}} \int \frac{1}{1+\exp \left(\frac{\varepsilon_{+}-\varepsilon_{F}}{k_{B} T}\right)} d \mathbf{k} .
$$


In the case of space homogeneous solutions, which will be considered for the determination of the mobilities, the Equation (1) becomes

$$
\frac{\partial f_{s}}{\partial \mathrm{t}}-\frac{e}{\hbar} \mathbf{E} \cdot \nabla_{\mathbf{k}} f_{s}=Q\left(f_{s}, f_{-s}\right) \quad(s= \pm 1)
$$

where $f_{s}$ depend only on $\mathrm{t}$ and $\mathbf{k}$.

In the valence band it is necessary to consider holes, instead of electrons, in order to avoid an infinite density of electrons. The distribution function of holes $f_{h}$ is related with $f_{-}$by

$$
f_{h}(\mathrm{t}, \mathbf{x}, \mathbf{k})=1-f_{-}(\mathrm{t}, \mathbf{x},-\mathbf{k})
$$

\section{The numerical method}

Since we expect an exponential decay of $f_{+}$and $1-f_{-}$, as $|\mathbf{k}| \rightarrow+\infty$, it is reasonable to choose a compact domain $\Omega \subseteq \mathbb{R}^{2}$, such that $f_{+}(t, \mathbf{k}) \approx 0$ and $1-f_{-}(t, \mathbf{k}) \approx 0$, for every $\mathbf{k} \notin \Omega$, and for every $\mathbf{x}$ and $t>0$.

Now we introduce a finite decomposition $\left\{C_{\alpha}: \alpha=1,2, \ldots, N\right\}$ of the domain $\Omega$, with the $C_{\alpha}$ 's open sets such that

$$
C_{\alpha} \subseteq \Omega \quad \forall \alpha, \quad C_{\alpha} \cap C_{\beta}=\emptyset \quad \forall \alpha \neq \beta, \quad \bigcup_{\alpha=1}^{N} \bar{C}_{\alpha}=\Omega
$$

We assume a constant approximation for each distribution function $f_{s}$ in every cell $C_{\alpha}$. If we denote by $\chi_{\alpha}$ the characteristic function relative to the cell $C_{\alpha}$, then

$$
f_{s}(\mathrm{t}, \mathbf{k}) \approx f_{s}^{\alpha}(\mathrm{t}), \forall \mathbf{k} \in C_{\alpha} \quad \Longleftrightarrow \quad f_{s}(\mathrm{t}, \mathbf{k}) \approx \sum_{\alpha=1}^{N} f_{s}^{\alpha}(\mathrm{t}) \chi_{\alpha}(\mathbf{k}), \forall \mathbf{k} \in \bigcup_{\alpha=1}^{N} C_{\alpha}
$$

A set of partial differential equations is now derived from the Boltzmann equations (1). The new unknowns are the $f_{s}^{\alpha}(\mathrm{t})$ 's instead of the distribution functions $f_{s}(\mathrm{t}, \mathbf{k})$. Formally integrating Equation (1) over each cell $C_{\alpha}$, we obtain

$$
\int_{C_{\alpha}} \frac{\partial f_{s}}{\partial \mathrm{t}} d \mathbf{k}-\int_{C_{\alpha}} \frac{e}{\hbar} \mathbf{E} \cdot \nabla_{\mathbf{k}} f_{s} d \mathbf{k}=\int_{C_{\alpha}} Q\left(f_{s}, f_{-s}\right) d \mathbf{k}
$$

The first integral of Equation (3) can be approximated easily, the integral involving the electric field is transformed by using the Gauss theorem. Of course, the result is strictly related to the geometry of the cells. We have used a reconstruction of the fluxes based on a Min-Mod slope limiter. The integral involving the collisional operator can be solved analytically after performing the discretization. For more details about the numerical method one can refer to [1,3] for the unipolar case and to [5] for the treatment of the coupled collisional terms in the bipolar case while all the details about the treatment of the drift term can be found in [3].

The spatial gradient $\nabla_{\mathbf{x}} f_{s}(\mathrm{t}, \mathbf{x}, \mathbf{k})$ can be discretized in the same way as the drift one. In this case the discretization depends on the geometry of the spatial domain and boundary conditions (see for instance $[6,7]$ ). Since in this paper we consider only space homogeneous solutions, the details are skipped.

The treatment of the term arising from the collision operator is straightforward. If $\mathbf{k} \in C_{\alpha}$, then we 
have

$$
\begin{aligned}
Q\left(f_{s}, f_{-s}\right)= & \sum_{s^{\prime}}\left[\left(1-f_{s}(\mathrm{t}, \mathbf{x}, \mathbf{k})\right) \int S_{s^{\prime}, s}\left(\mathbf{k}^{\prime}, \mathbf{k}\right) f_{s^{\prime}}\left(\mathrm{t}, \mathbf{x}, \mathbf{k}^{\prime}\right) d \mathbf{k}^{\prime}\right. \\
& \left.-f_{s}(\mathrm{t}, \mathbf{x}, \mathbf{k}) \int S_{s, s^{\prime}}\left(\mathbf{k}, \mathbf{k}^{\prime}\right)\left(1-f_{s^{\prime}}\left(\mathrm{t}, \mathbf{x}, \mathbf{k}^{\prime}\right)\right) d \mathbf{k}^{\prime}\right] \\
\approx & \sum_{s^{\prime}} \sum_{\beta=1}^{N}\left[\left(1-f_{s}^{\alpha}(\mathrm{t}, \mathbf{x})\right) \int_{C_{\beta}} S_{s^{\prime}, s}\left(\mathbf{k}^{\prime}, \mathbf{k}\right) f_{s^{\prime}}\left(\mathrm{t}, \mathbf{x}, \mathbf{k}^{\prime}\right) d \mathbf{k}^{\prime}\right. \\
& \left.-f_{s}^{\alpha}(\mathrm{t}, \mathbf{x}) \int_{C_{\beta}} S_{s, s^{\prime}}\left(\mathbf{k}, \mathbf{k}^{\prime}\right)\left(1-f_{s^{\prime}}\left(\mathrm{t}, \mathbf{x}, \mathbf{k}^{\prime}\right)\right) d \mathbf{k}^{\prime}\right] \\
\approx & \sum_{s^{\prime}} \sum_{\beta=1}^{N}\left[\left(1-f_{s}^{\alpha}(\mathrm{t}, \mathbf{x})\right) f_{s^{\prime}}^{\beta}(\mathrm{t}, \mathbf{x}) \int_{C_{\beta}} S_{s^{\prime}, s}\left(\mathbf{k}^{\prime}, \mathbf{k}\right) d \mathbf{k}^{\prime}\right. \\
& \left.-f_{s}^{\alpha}(\mathrm{t}, \mathbf{x})\left(1-f_{s^{\prime}}^{\beta}(\mathrm{t}, \mathbf{x})\right) \int_{C_{\beta}} S_{s, s^{\prime}}\left(\mathbf{k}, \mathbf{k}^{\prime}\right) d \mathbf{k}^{\prime}\right]
\end{aligned}
$$

So, defining

$$
A_{s, s^{\prime}}^{\alpha, \beta}=\int_{C_{\alpha}}\left[\int_{C_{\beta}} S_{s, s^{\prime}}\left(\mathbf{k}, \mathbf{k}^{\prime}\right) d \mathbf{k}^{\prime}\right] d \mathbf{k}
$$

it is immediate to verify that

$$
\int_{C_{\alpha}} Q\left(f_{s}, f_{-s}\right) d \mathbf{k} \approx \sum_{s^{\prime}} \sum_{\beta=1}^{N}\left[A_{s^{\prime}, s}^{\beta, \alpha}\left(1-f_{s}^{\alpha}(\mathrm{t}, \mathbf{x})\right) f_{s^{\prime}}^{\beta}(\mathrm{t}, \mathbf{x})-A_{s, s^{\prime}}^{\alpha, \beta} f_{s}^{\alpha}(\mathrm{t}, \mathbf{x})\left(1-f_{s^{\prime}}^{\beta}(\mathrm{t}, \mathbf{x})\right)\right] .
$$

In order to complete the numerical treatment of the Boltzmann equation it is necessary to specify the numerical domain in the $\mathbf{k}$-space. We choose the circle $|\mathbf{k}| \leq k_{\max }$, where $k_{\max }$ is a fixed maximum value such that $f_{+}$and $1-f_{-}$are negligible for all $\mathbf{k}$ such that $|\mathbf{k}|>k_{\max }$. The parameter $k_{\max }$ is checked a posteriori by means of numerical experiments.

We use modified polar coordinates by setting $\mathbf{k}=\sqrt{r}(\cos \vartheta, \sin \vartheta)$, and we introduce a regular decomposition. Hence the cell $C_{\alpha}$ is defined by the inequalities

$$
0 \leq r_{\alpha}<r<r_{\alpha}+\Delta r \leq \sqrt{k_{\max }} \quad \text { and } \quad 0 \leq \vartheta_{\alpha}<\vartheta<\vartheta_{\alpha}+\Delta \vartheta \leq 2 \pi,
$$

where $\Delta r$ and $\Delta \vartheta$ are constant for every cell $C_{\alpha}$.

The use of the variable $\sqrt{r}$ instead of the modulus of $\mathbf{k}$ is aimed to have integrable parameters arising from the force term of the Boltzmann equation. Now, one can obtain the explicit expression of the numerical parameters $A_{s, s^{\prime}}^{\alpha, \beta}$. The integrals involving the electric field are derived by means of the same technique (see [3]). More details about the collisional term of interband transtions can be found in [5].

We observe that considering the distribution $f_{h}$ of holes in the VB instead of the one of electrons, one gets that also $f_{h} \approx 0$ for all $\mathbf{k} \notin \Omega$, similarly to $f_{+}$, and this helps the numerical approach that we use for the integration of the transport equations.

From the approximation of the distribution functions, the average values of density $n$, velocity $\left\langle\mathbf{v}_{e}\right\rangle$ and energy $\left\langle\varepsilon_{e}>\right.$ for electrons are reconstructed as follows

$$
\begin{aligned}
& n(\mathrm{t}, \mathbf{x}):=\frac{2}{(2 \pi)^{2}} \int f_{+}(\mathrm{t}, \mathbf{x}, \mathbf{k}) d \mathbf{k} \approx \frac{2}{(2 \pi)^{2}} \sum_{\alpha=1}^{N} \operatorname{meas}\left(C_{\alpha}\right) f_{+}^{\alpha}(\mathrm{t}, \mathbf{x}), \\
& <\mathbf{v}_{e}>(\mathrm{t}, \mathbf{x}):=\frac{1}{n(\mathrm{t}, \mathbf{x})} \frac{2}{(2 \pi)^{2}} \int f_{+}(\mathrm{t}, \mathbf{x}, \mathbf{k}) \mathbf{v}_{+}(\mathbf{k}) d \mathbf{k} \approx \frac{1}{n(\mathrm{t}, \mathbf{x})} \frac{2}{(2 \pi)^{2}} \sum_{\alpha=1}^{N}\left[\int_{C_{\alpha}} \mathbf{v}_{+}(\mathbf{k}) d \mathbf{k}\right] f_{+}^{\alpha}(\mathrm{t}, \mathbf{x}),
\end{aligned}
$$




$$
<\varepsilon_{e}>(\mathrm{t}, \mathbf{x}):=\frac{1}{n(\mathrm{t}, \mathbf{x})} \frac{2}{(2 \pi)^{2}} \int f_{+}(\mathrm{t}, \mathbf{x}, \mathbf{k}) \varepsilon_{+}(\mathbf{k}) d \mathbf{k} \approx \frac{1}{n(\mathrm{t}, \mathbf{x})} \frac{2}{(2 \pi)^{2}} \sum_{\alpha=1}^{N}\left[\int_{C_{\alpha}} \varepsilon_{+}(\mathbf{k}) d \mathbf{k}\right] f_{+}^{\alpha}(\mathrm{t}, \mathbf{x}) .
$$

Similarly for the average values of density $p$, velocity $\left\langle\mathbf{v}_{h}>\right.$ and energy $<\varepsilon_{h}>$ for holes

$$
\begin{aligned}
& p(\mathrm{t}, \mathbf{x}):=\frac{2}{(2 \pi)^{2}} \int\left(1-f_{-}(\mathrm{t}, \mathbf{x},-\mathbf{k})\right) d \mathbf{k} \approx \frac{2}{(2 \pi)^{2}} \sum_{\alpha=1}^{N} \operatorname{meas}\left(C_{\alpha}\right)\left(1-f_{-}^{\alpha}(\mathrm{t}, \mathbf{x})\right) \\
&<\mathbf{v}_{h}>(\mathrm{t}, \mathbf{x}):=\frac{1}{p(\mathrm{t}, \mathbf{x})} \frac{2}{(2 \pi)^{2}} \int f_{h}(\mathrm{t}, \mathbf{x}, \mathbf{k}) \mathbf{v}_{h}(\mathbf{k}) d \mathbf{k}=\frac{1}{p(\mathrm{t}, \mathbf{x})} \frac{2}{(2 \pi)^{2}} \int\left(1-f_{-}(\mathrm{t}, \mathbf{x},-\mathbf{k})\right) \mathbf{v}_{h}(\mathbf{k}) d \mathbf{k} \\
&=\frac{1}{p(\mathrm{t}, \mathbf{x})} \frac{2}{(2 \pi)^{2}} \int\left(1-f_{-}(\mathrm{t}, \mathbf{x},-\mathbf{k})\right) \mathbf{v}_{+}(\mathbf{k}) d \mathbf{k} \\
& \approx-\frac{1}{p(\mathrm{t}, \mathbf{x})} \frac{2}{(2 \pi)^{2}} \sum_{\alpha=1}^{N}\left[\int_{C_{\alpha}} \mathbf{v}_{+}(\mathbf{k}) d \mathbf{k}\right]\left(1-f_{-}^{\alpha}(\mathrm{t}, \mathbf{x})\right) \\
&<\varepsilon_{h}>(\mathrm{t}, \mathbf{x}):=\frac{1}{p(\mathrm{t}, \mathbf{x})} \frac{2}{(2 \pi)^{2}} \int\left(1-f_{-}(\mathrm{t}, \mathbf{x},-\mathbf{k})\right) \varepsilon_{+}(\mathbf{k}) d \mathbf{k} \\
& \approx \frac{1}{p(\mathrm{t}, \mathbf{x})} \frac{2}{(2 \pi)^{2}} \sum_{\alpha=1}^{N}\left[\int_{C_{\alpha}} \varepsilon_{+}(\mathbf{k}) d \mathbf{k}\right]\left(1-f_{-}^{\alpha}(\mathrm{t}, \mathbf{x})\right)
\end{aligned}
$$

where we have used the property of the hole velocity: $\mathbf{v}_{h}(\mathbf{k})=\mathbf{v}_{+}(\mathbf{k})$. The above integrals are easily evaluated by taking into account that

$$
\begin{aligned}
& \operatorname{meas}\left(C_{\alpha}\right)=\frac{1}{2} \Delta r \Delta \vartheta \\
& \int_{C_{\alpha}} \mathbf{v}_{s} d \mathbf{k}=s v_{F} \Delta r\left(\cos \left(\vartheta+\frac{\Delta \vartheta}{2}\right) \sin \frac{\Delta \vartheta}{2}, \sin \left(\vartheta+\frac{\Delta \vartheta}{2}\right) \sin \frac{\Delta \vartheta}{2}\right), \\
& \int_{C_{\alpha}} \varepsilon_{s}(\mathbf{k}) d \mathbf{k}=\frac{1}{3} s v_{F} \hbar \Delta \vartheta\left[\sqrt{\left(r_{\alpha}+\Delta r\right)^{3}}-\sqrt{\left(r_{\alpha}\right)^{3}}\right] .
\end{aligned}
$$

Regarding the total current density $-e \mathbf{J}$, it is given by the sum of the contribution from electrons in the CB band and holes in the VB

$$
\mathbf{J}=\mathbf{J}_{n}-\mathbf{J}_{p}
$$

The term $\mathbf{J}_{n}$ is given by $n<\mathbf{v}_{e}>$ while

$$
\mathbf{J}_{p}=\frac{2}{(2 \pi)^{2}} \int\left(1-f_{-}(\mathrm{t}, \mathbf{x},-\mathbf{k})\right) \mathbf{v}_{h}(\mathbf{k}) d \mathbf{k}=-\frac{2}{(2 \pi)^{2}} \int\left(1-f_{-}(\mathrm{t}, \mathbf{x}, \mathbf{k})\right) \mathbf{v}_{h}(\mathbf{k}) d \mathbf{k} .
$$

For other details about the model see [5].

\section{The mobility model: the case of suspended monolayer graphene}

The direct solution of the Boltzmann equation, even though highly accurate, requires computational times too long for a practical use in electronic CAD. For applications in electronic device modeling, in order to reduce the computational effort, macroscopical models are mostly adopted. The simplest and most popular ones are the drift-diffusion (DD) models. They are constituted by two coupled nonlinear diffusion equations for the electron density $n$ and the hole density $p$

$$
\begin{aligned}
& \frac{\partial n}{\partial t}+\nabla \cdot \mathbf{J}_{n}=-R+G \\
& \frac{\partial p}{\partial t}+\nabla \cdot \mathbf{J}_{p}=-R+G,
\end{aligned}
$$


where

$$
\begin{aligned}
& \mathbf{J}_{n}=-\mu_{n}\left(U_{T} \nabla n-n \nabla \phi\right) \\
& \mathbf{J}_{p}=-\mu_{p}\left(U_{T} \nabla p+p \nabla \phi\right)
\end{aligned}
$$

are the electron and hole currents divided by the elementary charge (- $e$ for electrons and $e$ for holes), with $U_{T}=k_{B} T / e$ the thermal potential, $k_{B}$ being the Boltzmann constant, $T$ the constant lattice temperature, $e$ the positive elementary charge. $\mu_{n}$ and $\mu_{p}$ are the mobilities for electrons and holes respectively while $R$ and $G$ are the recombination and generation terms. The function $\phi(x)$ is the electric potential which solves the Poisson equation. Of course, in the case of a constant electric field, due to $\mathbf{E}=-\nabla \phi$, we have that $\phi(x)$ is linear.

The crucial issue is to devise appropriate models for the mobilities and the recombination-generation terms. However, usually the characteristic times of the recombination-generation terms are much longer than the typical evolution times. Moreover, for a Fermi energy greater than $0.2 \mathrm{eV}, R-G$ is essentially negligible because the Pauli exclusion principle prevents the formation of electron-hole couples. For a Fermi energy less than - $0.2 \mathrm{eV}$ practically there are not electrons around the Dirac point and again $R-G$ is essentially negligible. Therefore, as first instance, it is more important, to model only the mobilities, neglecting the electron-hole coupling.

The mobilities are definite through the relations (in the one dimensional case)

$$
v_{n}=\mu_{n}(E, n) E, \quad v_{h}=\mu_{p}(E, p) E,
$$

where $E, v_{n}$ and $v_{p}$ are the significant components of the the electric field, the average electron velocity and the average hole velocity. The velocities are related to the currents as follows

$$
v_{n}=J_{n} / n, \quad v_{h}=J_{p} / p .
$$

First we analyze the low field mobility $\mu_{0}$, that is defined as

$$
\mu_{0}(n)=\lim _{E \rightarrow 0} \mu_{n}(E, n) .
$$

In principle the low field mobilities of electron and holes are different. In suspended graphene they take the same values.

The main concern regarding the low field mobility is that its determination from experimental data is rather cumbersome on account of the intrinsic statistical noise. A similar issue arises if the results obtained by DSMC are employed. The use of the results given by the DG approach avoids such difficulties because reduces (practically avoids) the effects of the statistical noise.

For the determination of the mobility curves it is enough to consider homogeneous solutions. In this section we examine the case of a suspended single sheet of graphene, as reported in Figure 1. We suppose that the contacts are deeply infinite and the potential is constant in each of them. Therefore, the electric field is constant and transversal with respect to the contact. Thus the system can be solved in the interval $[0, L]$ with Dirichlet boundary conditions

$$
\left.n\right|_{x=0}=n_{0},\left.\quad n\right|_{x=L}=n_{L},\left.\quad p\right|_{x=0}=p_{0},\left.\quad p\right|_{x=L}=p_{L} .
$$

The solution does not depend on space.

For several values of $n$ corresponding to a Fermi level between $0 \mathrm{eV}$ and $0.4 \mathrm{eV}$, the low field mobility is extrapolated, by a linear regression model, computing the mobility at different low values of electric field, between $0.0005 \mathrm{~V} / \mu \mathrm{m}$ and $0.0025 \mathrm{~V} / \mu \mathrm{m}$, by using the values of the currents obtained by a direct solution of the Boltzmann equation with the DG method outlined in Section 3. One observes (see Figure 2) that the low field mobility behaviour versus the density (or equivalently the Fermi energy) is similar to the log-normal distribution and therefore we assume the following expression

$$
\mu_{0}(n)=\tilde{\mu}_{0} \frac{\exp \left(-\frac{\left(\log \left(n / n_{\text {ref }}\right)-m\right)^{2}}{2 \sigma^{2}}\right)}{\sqrt{2 \pi} \sigma n / n_{\text {ref }}},
$$




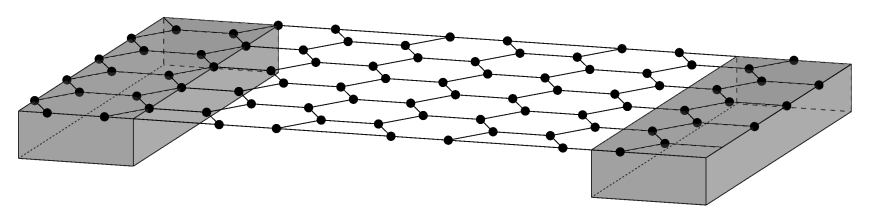

Figure 1. Representation of a suspended graphene sheet with contacts.

where $\tilde{\mu}_{0}, n_{r e f}, m$ and $\sigma$ are fitting parameters. The behaviour is the same for holes on account of the symmetry between the hole and electron distributions.

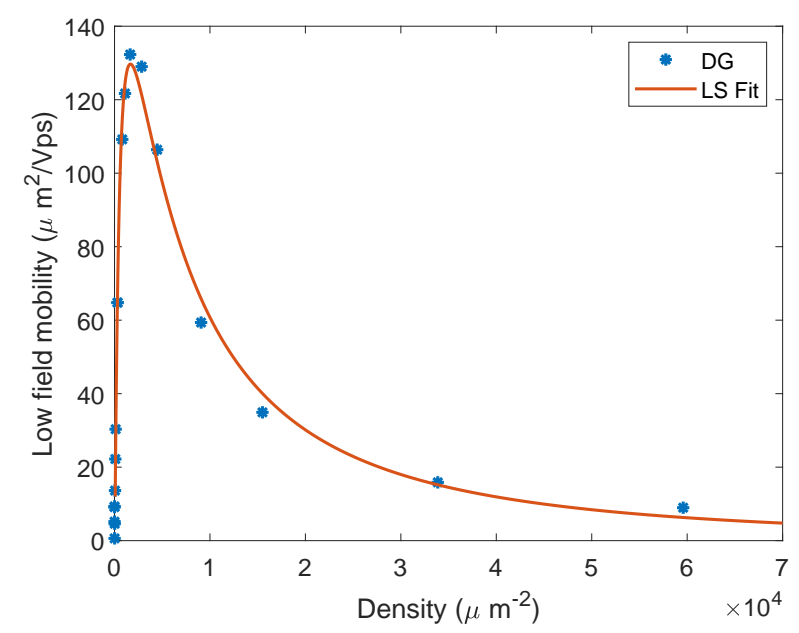

Figure 2. Comparison between the low field mobility simulated with the DG method and the fitted one.

We have estimated the parameters by means of the least squares method. The obtained values are reported in Table 1.

Table 1. Low field mobility parameters.

\begin{tabular}{c|c|c|c}
$\tilde{\mu}_{0}$ & $n_{\text {ref }}$ & $m$ & $\sigma$ \\
\hline $217.07 \mu \mathrm{m}^{2} / \mathrm{V} \mathrm{ps}$ & $8951.4 \mu \mathrm{m}^{-2}$ & -0.0027671 & 1.3423 \\
\hline
\end{tabular}

As second step, we include the electric field dependence by proposing the same model of Ref. [2]

$$
\mu(E, n)=\frac{\mu_{0}(n)+\frac{v_{F}}{E}\left(\frac{E}{E_{r e f}}\right)^{\beta_{1}}}{1+\left(\frac{E}{E_{r e f}}\right)^{\beta_{2}}+\gamma\left(\frac{E}{E_{r e f}}\right)^{\beta_{3}}},
$$

where $E_{r e f}, \beta_{1}, \beta_{2}, \beta_{3}$ and $\gamma$ are fitting parameters. In this case we evaluated the mobility by means of the DG results of the transport equations at different densities, corresponding to the Fermi levels of 0.2 $\mathrm{eV}, 0.3 \mathrm{eV}, 0.4 \mathrm{eV}$, and for a range of electric fields between $0.025 \mathrm{~V} / \mu \mathrm{m}$ and $10 \mathrm{~V} / \mu \mathrm{m}$. The obtained parameters are reported in Table 2. A comparison between fitted and simulated values of mobility models

Table 2. Mobility model parameters.

\begin{tabular}{c|c|c|c|c}
$E_{r e f}$ & $\beta_{1}$ & $\beta_{2}$ & $\beta_{3}$ & $\gamma$ \\
\hline $0.0421 \mathrm{~V} / \mu \mathrm{m}$ & 1.2898 & 1.4050 & 0.9627 & 5.0918 \\
\hline
\end{tabular}

and corresponding current densities are plotted in Figure 3 

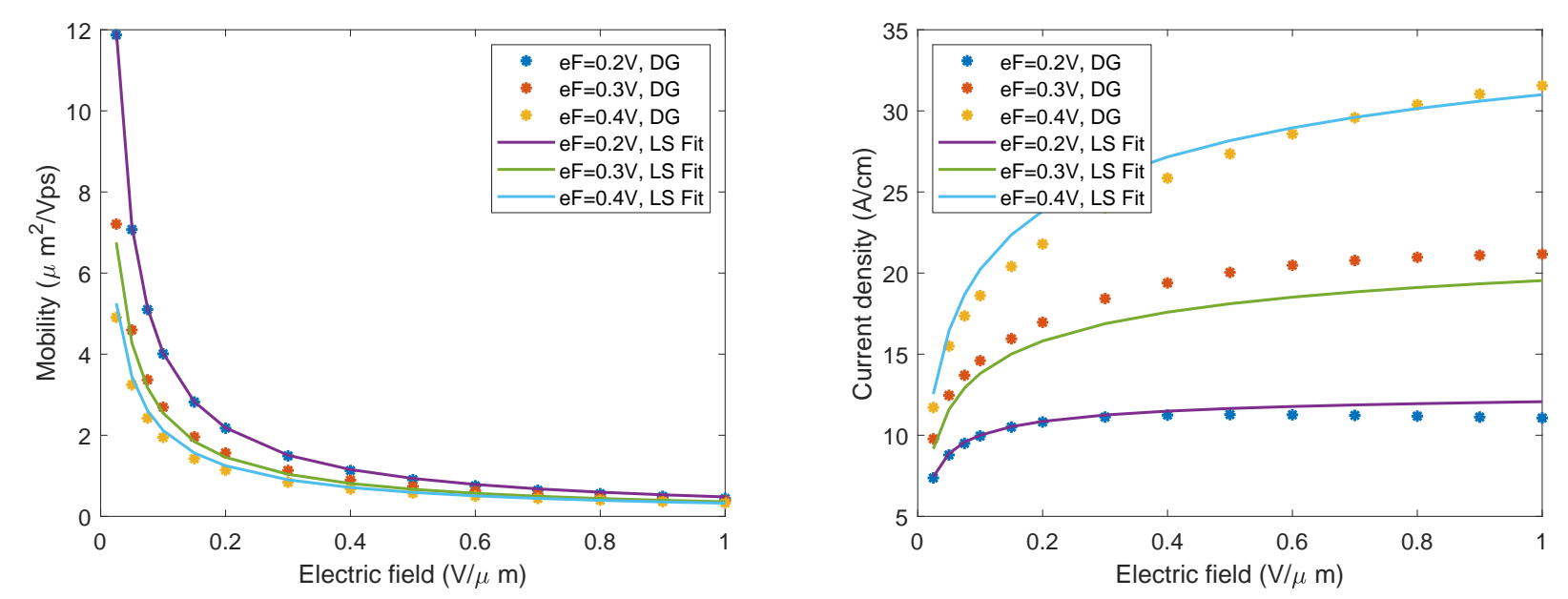

Figure 3. On the left comparison between the mobility simulated with the DG method and the fitted one. On the right the comparison of the simulated and fitted current densities for several values of the Fermi energy.

\section{Comparison with other mobility models}

There are available in the literature several expressions of the mobilities. Most of them are based on heuristic considerations or experimental data. A widely used model is that of Dorgan et al. [8]

$$
\mu(x)=\frac{\nu}{\left[1+\left(\nu E / v_{s a t}\right)^{\gamma}\right]^{1 / \gamma}},
$$

where $v_{\text {sat }}$ is the saturation velocity, $\gamma \approx 2$ and

$$
\nu(x)=\frac{\mu_{0}}{\left(1+n / n_{r e f}\right)^{\alpha}},
$$

with $\mu_{0}$ the low field mobility, $n_{\text {ref }}=1.1 \times 10^{5} \mu \mathrm{m}^{-2}$ and $\alpha=2.2$.

In [8] the value $\mu_{0}=0.4650 \mu \mathrm{m}^{2} / \mathrm{V}$ ps is taken while the saturation velocity is evaluated as

$$
v_{s a t}=\frac{2}{\pi} \frac{\omega_{O P}}{\sqrt{\pi n}} \sqrt{1-\frac{\omega_{O P}^{2}}{4 \pi n v_{F}^{2}}} \frac{1}{N_{O P}+1},
$$

where $\hbar \omega_{O P}$ is the energy of optical phonons, that here takes the value of $160 \mathrm{meV}$, and

$$
N_{O P}=\frac{1}{\exp \left(\frac{\hbar \omega_{O P}}{k_{B} T}\right)-1}
$$

is the phonon occupation number. The previous expressions, written for the electron density $n$, are still valid for the hole density $p$. Here for the saturation velocity the values reported in Table 3 have been adopted.

Table 3. Adopted saturation velocity values.

\begin{tabular}{c|c}
$e F$ & $v_{\text {sat }}$ \\
\hline $0.2 \mathrm{eV}$ & $0.5841 \mu \mathrm{m} / \mathrm{ps}$ \\
$0.3 \mathrm{eV}$ & $0.4393 \mu \mathrm{m} / \mathrm{ps}$ \\
$0.4 \mathrm{eV}$ & $0.3426 \mu \mathrm{m} / \mathrm{ps}$ \\
\hline
\end{tabular}

In Ref. [2] the authors used the model given by the equation (9) but with a different expression of the low field mobility

$$
\mu_{0}=\mu_{0}(n)=\frac{\tilde{\mu}}{1+\left(n / n_{r e f}\right)^{\alpha}},
$$


(a similar expression can be introduced also for holes) with $n_{\text {ref }}=0.5 \times 10^{4} \mu \mathrm{m}^{-2}, \alpha=1.2916$ and $\tilde{\mu}=2 \mu_{0}\left(n_{\text {ref }}\right)=373.4306 \mu \mathrm{m}^{2} / \mathrm{V}$ ps. The reader is referred to [2] for the specific values of the parameters.

In Figure 4 we have compared the different mobility curves among them and with the results of the direct solution of the transport equations in the case of a Fermi energy $0.2 \mathrm{eV}$ (top left). In the figure we have also included a modified Dorgan model (MDM) where the low field mobility is taken as that given by relation (8).

The model of Dorgan et al. underestimates the mobility, in particular for low fields. MDM and the model in [2] are in good agreement between them but there is a considerable discrepancy with the results given by the Boltzmann equation. Apparently the inclusion of the interband scattering, neglected in [2], has a relevant role for a correct determination of the low field mobility. At high fields all the mobility curves become closer.

In Figure 4 (top right and bottom) Fermi energies of $0.3 \mathrm{eV}$ and $0.4 \mathrm{eV}$ are considered. In these plots the original model of Dorgan et al. is not reported because of the high discrepancy. In the case of a Fermi energy of $0.3 \mathrm{eV}$ the model in [2] is more accurate than MDM for low fields. In the case of a Fermi energy of $0.4 \mathrm{eV}$ the model in [2] is more accurate than MDM for low fields but less accurate for high fields.
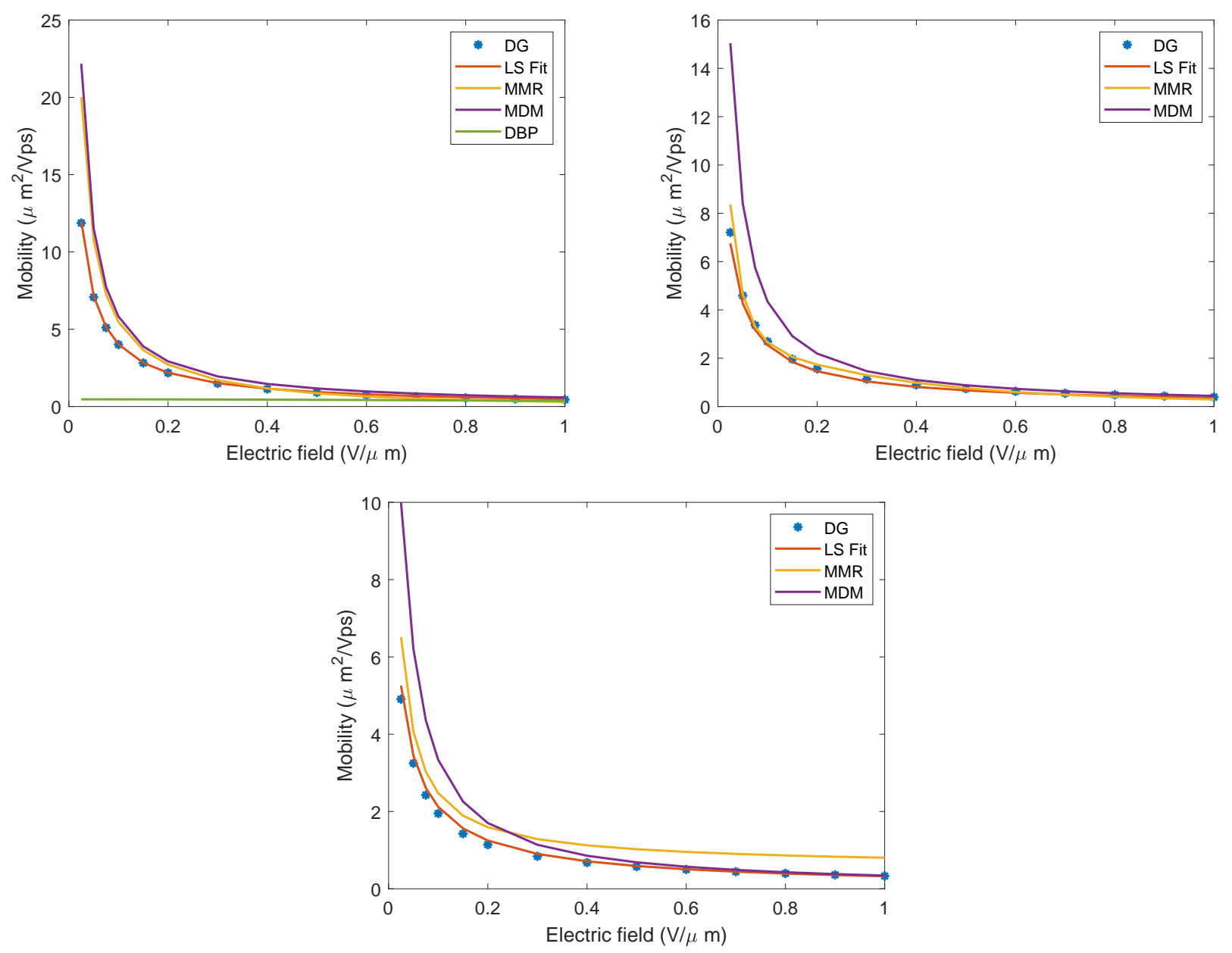

Figure 4. Comparison among the mobility models of Ref. [2] (MMR) and Ref. [8] (MDM) and the fitted one with respect to the DG simulations at a Fermi level of $0.2 \mathrm{eV}$ (top left), $0.3 \mathrm{eV}$ (top right) and $0.4 \mathrm{eV}$ (bottom). At the Fermi level of $0.2 \mathrm{eV}$ it is also shown the original model of Ref. [8] (DBP).

\section{The mobility model: the case of graphene on substrate}

In order to include the presence of a substrate as depicted in Figure 5, a further generalization of the mobility model is required. In this situation usually one observes a degradation of the current due to the 
additional scatterings of the charges in the graphene with the phonons and impurities in the substrate. We have considered the case of graphene on $\mathrm{SiO}_{2}$ (silicon dioxide) and again used a DG method for solving the transport equations (the interested reader is referred to [3,9] for details).

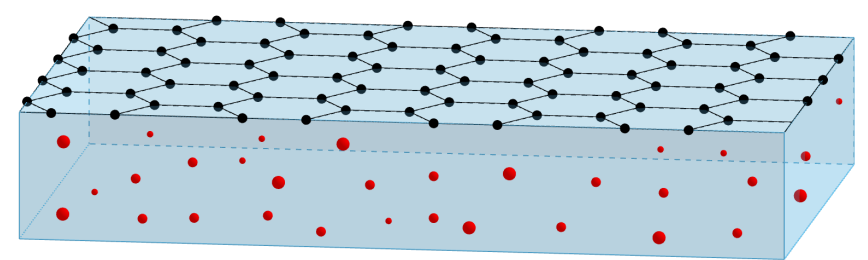

Figure 5. Schematic representation of a graphene sheet on a substrate. The dots stand for the impurities in the oxide.

A crucial parameter, entering the expression of the collision term, is the average distance $d$ between the graphene and the impurities. The value of $d$ is considered to lie in the range between 0 and $1 \mathrm{~nm}$. We have obtained the mobility curves for some values of $d$ by using again relations (8) and (9).

The fitted parameters are reported in Table 4. The obtained mobilities and current densities are shown in Figures 6,7 and 8 considering $d=0,0.5,1 \mathrm{~nm}$ and several values of the Fermi energy.

Table 4. Mobility model parameters on $\mathrm{SiO}_{2}$.

\begin{tabular}{c|c|c|c|c|c}
$d$ & $E_{\text {ref }}$ & $\beta_{1}$ & $\beta_{2}$ & $\beta_{3}$ & $\gamma$ \\
\hline $0 \mathrm{~nm}$ & $0.4879 \mathrm{~V} / \mu \mathrm{m}$ & 2.0736 & 0.8672 & 1.6147 & 4.9884 \\
$0.5 \mathrm{~nm}$ & $0.3875 \mathrm{~V} / \mu \mathrm{m}$ & 1.9923 & 2.3816 & 1.2336 & 4.8663 \\
$1 \mathrm{~nm}$ & $0.3626 \mathrm{~V} / \mu \mathrm{m}$ & 1.8753 & 2.0545 & 1.1854 & 5.6979 \\
\hline
\end{tabular}

Table 5. Low field mobility on $\mathrm{SiO}_{2}$.

\begin{tabular}{c|c|c|c}
$d$ & $\mu_{0}(e F=0.2 \mathrm{eV})$ & $\mu_{0}(e F=0.3 \mathrm{eV})$ & $\mu_{0}(e F=0.4 \mathrm{eV})$ \\
\hline $0 \mathrm{~nm}$ & $2.867 \mu \mathrm{m}^{2} / \mathrm{V} \mathrm{ps}$ & $1.252 \mu \mathrm{m}^{2} / \mathrm{V} \mathrm{ps}$ & $0.676 \mu \mathrm{m}^{2} / \mathrm{V} \mathrm{ps}$ \\
$0.5 \mathrm{~nm}$ & $4.106 \mu \mathrm{m}^{2} / \mathrm{V} \mathrm{ps}$ & $2.123 \mu \mathrm{m}^{2} / \mathrm{V} \mathrm{ps}$ & $1.353 \mu \mathrm{m}^{2} / \mathrm{V} \mathrm{ps}$ \\
$1 \mathrm{~nm}$ & $5.704 \mu \mathrm{m}^{2} / \mathrm{V} \mathrm{ps}$ & $3.345 \mu \mathrm{m}^{2} / \mathrm{V} \mathrm{ps}$ & $2.366 \mu \mathrm{m}^{2} / \mathrm{V} \mathrm{ps}$ \\
\hline
\end{tabular}

As expected we have a degradation of the mobility which reduces by increasing $d$. The latter effect is easily explained because there is a reduction of the scattering rate with impurities as the distance increases between the graphene sheet and the impurities in the oxide.
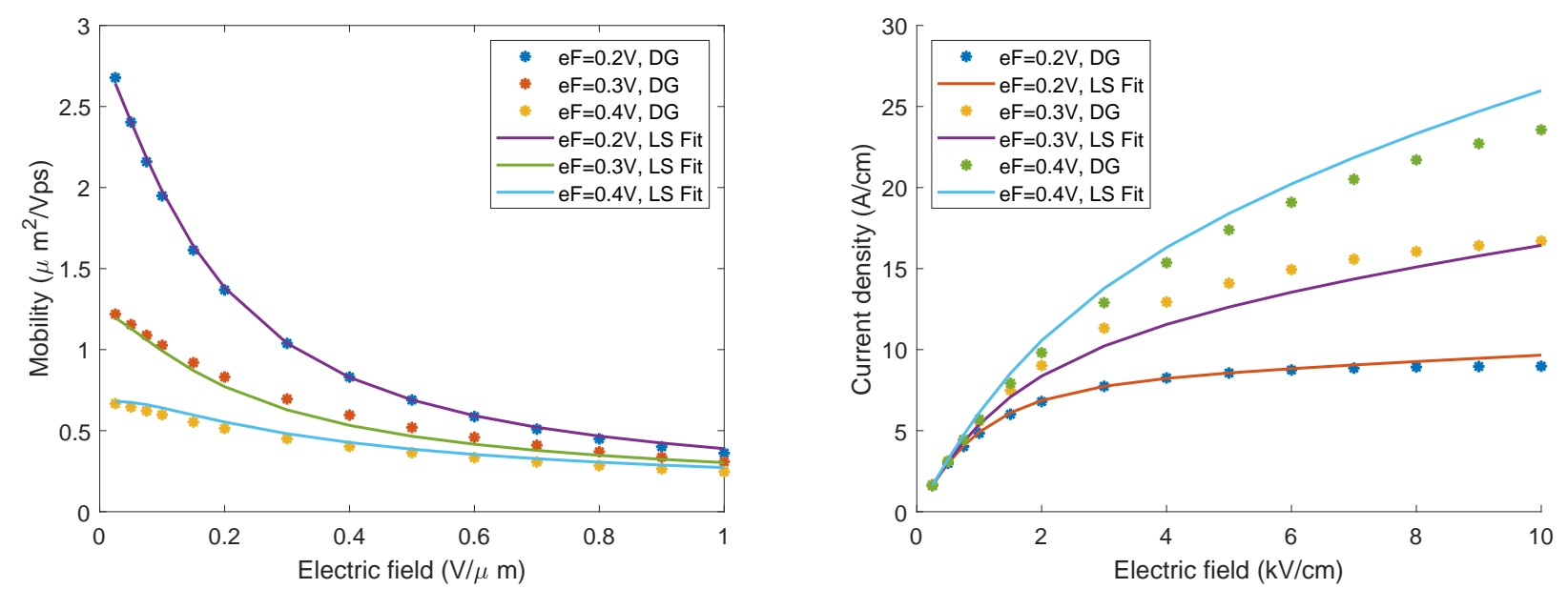

Figure 6. On the left comparison between the simulated by DG and the fitted mobility models in the case of graphene on $\mathrm{SiO}_{2}$ with a distance between the graphene sheet and impurities of $d=0 \mathrm{~nm}$. On the right the same for the densities. 

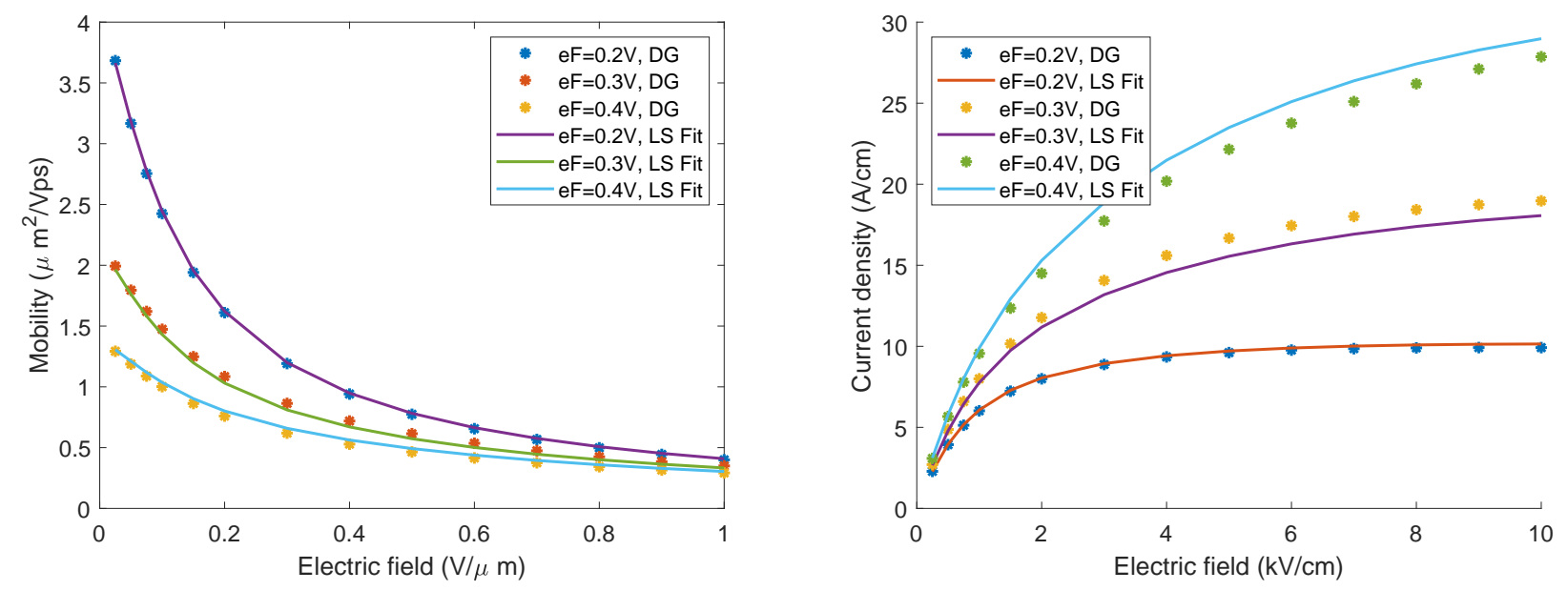

Figure 7. On the left comparison between the simulated by DG and the fitted mobility models in the case of graphene on $\mathrm{SiO}_{2}$ with a distance between the graphene sheet and impurities of $d=0.5 \mathrm{~nm}$. On the right the same for the densities.
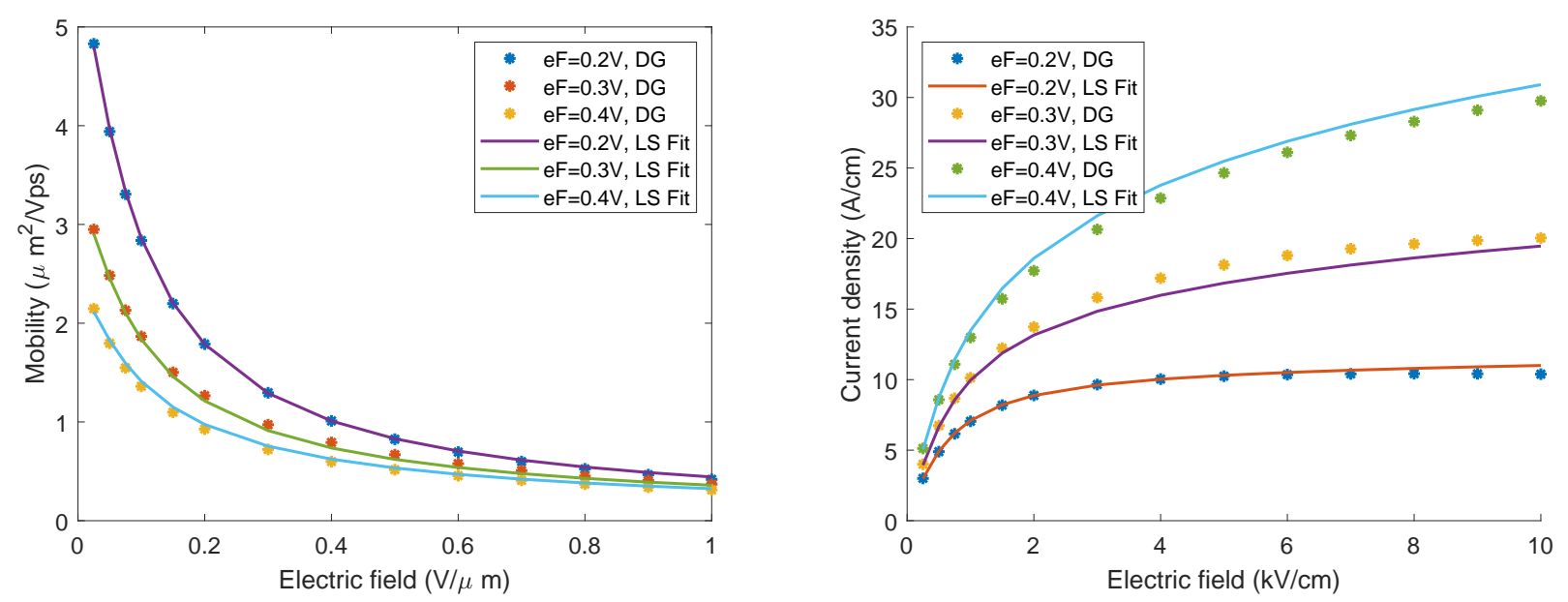

Figure 8. On the left comparison between the simulated by DG and the fitted mobility models in the case of graphene on $\mathrm{SiO}_{2}$ with a distance between the graphene sheet and impurities of $d=1 \mathrm{~nm}$. On the right the same for the densities.

\section{Conclusions}

New mobility models for charge transport in graphene have been obtained by resorting to an extensive numerical simulation of the Boltzmann transport equations with a DG method. Both the cases of suspended graphene and graphene on substrate have been considered. Comparisons with other models present in the literature show a considerable improvement.

Further developments could include the effects of crystal heating and quantum effects in the mobility models. Although the subject is still in an early stage, attempts in this direction can be found in $[10,11]$ regarding the thermal influence on the electric performance, and in [12-15] regarding the inclusion of quantum corrections.

\section{Acknowledgments}

The author V. R. acknowledges the financial support by the project FIR 2016-2018 Modellistica, simulazione e ottimizzazione del trasporto di cariche in strutture a bassa dimensionalità, University of Catania. The author G. N. acknowledges the financial support by the project FIR 2016-2018 Metodi gruppali e umbrali per modelli di diffusione e trasporto, University of Catania. Both authors acknowledge INDAM (GNFM) and Progetto Galileo 2018 Modelli cinetici classici e quantistici e loro limiti 
idrodinamici: aspetti tecnici e applicativi.

\section{References}

1. V. Romano, A. Majorana, and M. Coco, DSMC method consistent with the Pauli exclusion principle and comparison with deterministic solutions for charge transport in graphene, Journal of Computational Physics, vol. 302, pp. 267-284, 2015.

2. A. Majorana, G. Mascali, and V. Romano, Charge transport and mobility in monolayer graphene, $J$. Math. Industry, no. 7:4, doi:10.1186/s13362-016-0027-3, 2016.

3. M. Coco, A. Majorana, and V. Romano, Cross validation of discontinuous Galerkin method and Monte Carlo simulations of charge transport in graphene on substrate, Ricerche di matematica, vol. 66, pp. 201-220, 2017.

4. A. H. C. Neto, F. Guinea, N. M. R. Peres, K. S. Novoselov, and A. K. Geim, The electronic properties of graphene, Reviews of Modern Physics, vol. 81, pp. 109-162, 2009.

5. A. Majorana, G. Nastasi, and V. Romano, Simulation of bipolar charge transport in graphene by using a discontinuous Galerkin method, Communications in Computational Physics, vol. 26, no. 1, pp. 114-134, 2019.

6. Y. Cheng, I. M. Gamba, A. Majorana, and C.-W. Shu, A discontinuous Galerkin solver for BoltzmannPoisson systems in nano devices, Comput. Methods Appl. Mech. Engrg., vol. 198, no. 37-40, pp. 3130$3150,2009$.

7. Y. Cheng, I. M. Gamba, A. Majorana, and C.-W. Shu, A brief survey of the discontinuous Galerkin method for the Boltzmann-Poisson equations, Boletin de la Sociedad Espanola de Matematica Aplicada, vol. 54, pp. 47-64, 2011.

8. V. E. Dorgan, M.-H. Bae, and E. Pop, Mobility and saturation velocity in graphene on SiO2, Appl. Phys. Lett., vol. 97, p. 082112, 2010.

9. M. Coco, A. Majorana, G. Nastasi, and V. Romano, High-field mobility in graphene on substrate with a proper inclusion of the Pauli exclusion principle, Atti dell'Accademia Peloritana dei Pericolanti, in press.

10. G. Mascali and V. Romano, Charge transport in graphene including thermal effetcs, SIAM J. Appl. Mathematics, vol. 77, no. 2, pp. 593-613, 2017.

11. M. Coco and V. Romano, Simulation of Electron-Phonon Coupling and Heating Dynamics in Suspended Monolayer Graphene Including All the Phonon Branches, J. Heat Transfer, vol. 140, p. 092404, 2018.

12. O. Morandi, Wigner model for quantum transport in graphene, J. Phys. A: Math. Theor., vol. 44, p. 265301, 2011.

13. L. Barletti, Hydrodynamic equations for electrons in graphene obtained from the maximum entropy principle, J. Math. Phys., vol. 55, no. 8, p. 083303, 2014.

14. O. Muscato, A benchmark study of the Signed-particle Monte Carlo algorithm for the Wigner equation, Comm. in Appl. and Industrial Math., vol. 8, no. 1, pp. 237-250, 2017.

15. L. Luca and V. Romano, Quantum corrected hydrodynanic models for charge transport in graphene, Preprint, 2018. 